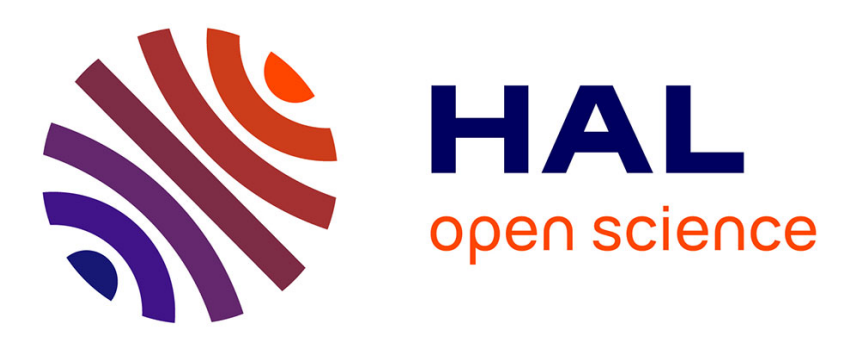

\title{
Asservissement de la fréquence d'un laser à colorant sur une référence atomique balayable par un champ magnétique
}

\author{
N. Courtier, M. Dumont
}

\section{- To cite this version:}

N. Courtier, M. Dumont. Asservissement de la fréquence d'un laser à colorant sur une référence atomique balayable par un champ magnétique. Revue de Physique Appliquée, 1981, 16 (10), pp.601610. 10.1051/rphysap:019810016010060100 . jpa-00244953

HAL Id: jpa-00244953

https://hal.science/jpa-00244953

Submitted on 1 Jan 1981

HAL is a multi-disciplinary open access archive for the deposit and dissemination of scientific research documents, whether they are published or not. The documents may come from teaching and research institutions in France or abroad, or from public or private research centers.
L'archive ouverte pluridisciplinaire HAL, est destinée au dépôt et à la diffusion de documents scientifiques de niveau recherche, publiés ou non, émanant des établissements d'enseignement et de recherche français ou étrangers, des laboratoires publics ou privés. 


\title{
Asservissement de la fréquence d'un laser à colorant sur une référence atomique balayable par un champ magnétique
}

\author{
N. Courtier et M. Dumont \\ Laboratoire de Physique des Lasers, Université Paris-Nord, avenue J.-B.-Clément, 93430 Villetaneuse, France \\ (Reçu le 15 juin 1981, accepté le 10 juillet 1981)
}

\begin{abstract}
Résumé. - Pour balayer de façon reproductible, à au moins un mégahertz, la fréquence d'un laser à colorant sur une très large plage ( 3 ou $4 \mathrm{GHz}$ ), nous avons verrouillé la fréquence du faisceau émis sur une raie d'absorption saturée du néon balayée par effet Zeeman (lumière polarisée $\sigma$ ). Le montage nécessite plusieurs boucles d'asservissements.
\end{abstract}

\begin{abstract}
To scan the frequency of the dye laser over a very large range ( 3 to $4 \mathrm{GHz}$ ) with a reproducibility of at least one megahertz, the frequency of the emitted beam is locked to a neon satured absorption line scanned by Zeeman effect ( $\sigma$ polarized light). The configuration requires several servo-loops.
\end{abstract}

Pour répondre efficacement aux exigences de la spectroscopie à haute résolution, le laser à colorant doit être, non seulement accordable sur une large plage de fréquences, mais présenter une excellente stabilité tant sur la fréquence sélectionnée que sur l'intensité du faisceau émis.

Il nous fallait répondre aux exigences d'une expérience d'absorption saturée à deux lasers, un laser pompe, un laser sonde. Cette expérience nécessite donc des sources stabilisées au mieux en intensité, de puissance suffisante (de l'ordre de $100 \mathrm{~mW}$ pour le faisceau pompe) et de fréquences définies de façon absolue à mieux qu'un mégahertz et balayable sur les quelques gigahertz du profil Doppler de la raie à étudier. Parmi les différentes techniques qui permettent une excursion en fréquence plus ou moins grande autour d'une valeur connue, nous citerons les deux exemples suivants :

- dans la première méthode, la fréquence du laser est asservie sur un interféromètre Fabry-Perot balayable (à l'aide d'une céramique piézo-électrique, une lame à commande galvanométrique ou par variation de la pression). Le balayage de la fréquence est contrôlé par observation, à côté du signal physique étudié, des franges d'un Fabry-Perot long (intervalle spectral libre petit) et très stable.

Cette méthode permet l'enregistrement d'un spectre sur une très grande plage de fréquence (supérieure à $10 \mathrm{GHz}$ ). Il faut que le spectre contienne une raie connue par rapport à laquelle tous les écarts de fréquence sont mesurés en se servant des pics du Fabry-Perot de référence comme d'une règle graduée. Cependant, avec cette méthode, il est difficile de caler longtemps la fréquence sur une valeur déterminée ou de réaliser plusieurs balayages consécutifs parfaitement identiques (pour moyenner le signal par exemple).

- la seconde méthode utilisée couramment en spectroscopie à très haute résolution [1] ou en métrologie [2] est celle du "décalage en fréquence" de deux lasers. Pour cela on verrouille la fréquence du laser $n^{0} 1$ (laser de référence) sur un signal d'absorption saturée (référence atomique absolue) et l'on pilote la fréquence du $n^{\circ} 2$ grâce au signal de battement entre les deux lasers. La fréquence de battement peut simplement être mesurée à l'aide d'un fréquencemètre, elle peut également être asservie sur un oscillateur RF de fréquence fixe ou balayable. Cette méthode est certainement la meilleure en spectroscopie moléculaire à très haute résolution, cependant en plus du fait qu'elle nécessite deux lasers, elle est d'usage très limité en spectroscopie atomique car l'amplitude de balayage continu est limitée à quelques centaines de mégahertz.

Nous avons retenu une troisième solution, qui, tout en n'utilisant qu'un seul laser, permet à la fois une excursion large et déterminée de la fréquence autour de la raie atomique à étudier. Pour cela, nous utilisons comme référence, le signal d'absorption saturée obtenu en lumière polarisée circulairement, 
et le décalage est obtenu en appliquant un champ magnétique à la cellule d'absorption. Dans le cas d'une structure Zeeman simple

$$
\left(J_{\mathrm{a}}=0, J_{\mathrm{b}}=1 \quad \text { ou } \quad J_{\mathrm{a}}=J_{\mathrm{b}}=1 / 2 \quad \text { ou } \quad g_{\mathrm{a}}=g_{\mathrm{b}}\right)
$$

c'est-à-dire dans le cas où il n'y a qu'une seule composante $\sigma^{+}$(et une composante $\sigma^{-}$) on peut balayer continûment la fréquence sur une plage de $\pm 1,5$ à $2 \mathrm{GHz}$ autour du centre de la raie atomique pour un balayage du champ magnétique de l'ordre de $\pm 1 \mathrm{kG}$ (le facteur de Landé, $g$, étant le plus souvent compris entre 1 et 1,5). L'écart de fréquence est alors connu et reproductible avec la précision du champ magnétique.

Lorsque la structure Zeeman de la raie est plus compliquée, la présence de plusieurs composantes $\sigma^{+}$ (ou $\sigma^{-}$) ne permet plus un balayage continu de la fréquence. Ce balayage est possible dans une petite zone, près du centre de la raie, lorsque les différentes composantes $\sigma^{+}$ne sont pas résolues, ou, au contraire loin du centre lorsqu'elles sont totalement résolues et que l'on peut choisir l'une ou l'autre d'entre elles. Si un balayage large est nécessaire, il est alors obligatoire d'utiliser deux lasers : par exemple, il est possible de stabiliser le laser sur un Fabry-Perot de référence, celui-ci étant accroché sur un second laser de référence verrouillé sur une raie $J=0, J=1$ balayée magnétiquement par notre méthode.

Dans les lasers à colorant continus, le milieu amplificateur (constitué d'un jet de colorant) est une source de bruit importante, difficilement contrôlable, qui génère des fluctuations tant sur l'amplitude du faisceau que sur sa fréquence (jitter libre de quelques mégahertz et souvent de quelques dizaines de mégahertz). Il est donc nécessaire de stabiliser ces lasers avec des servo-mécanismes ayant une grande dynamique et une large bande passante. Ceci ne peut être réalisé par asservissement direct sur une raie d'absorption saturée en raison du bruit qui l'affecte. Ce bruit est particulièrement important dans notre cas, car nous travaillons sur des raies d'absorption issues d'un niveau métastable du néon et qui nécessitent la présence d'une décharge dans le gaz. Pour ne pas transférer, à la fréquence du laser, le bruit lié à la décharge, il est nécessaire d'intégrer le signal d'absorption saturée sur un temps de l'ordre de la seconde : l'asservissement piloté par le pic d'absorption saturée a donc, au plus, une bande passante de l'ordre de $1 \mathrm{~Hz}$. Pour corriger tout le bruit haute fréquence il faut donc préstabiliser le laser sur un Fabry-Perot de référence (cavité passive, donc très stable à court terme) et utiliser le signal d'absorption saturée pour éliminer les dérives thermiques lentes du Fabry-Perot.

Nous décrirons tout d'abord ( $(1$.) la réalisation du laser monomode, préstabilisé en fréquence et en amplitude, puis $(\S 2$.) nous l'asservirons sur une référence atomique balayable par un champ magnétique.
1. Lasers à colorant monomodes préstabilisés. Nous disposions de deux lasers à colorant :

- le modèle 580 A de Spectra-Physics (580 A S.P.)

- le modèle 590 de Cohérent Radiation (590 C.R.).

Un seul de ces lasers était monomode de construction (580 A S.P.), nous avons rendu le second (590 C.R.) monomode en plaçant dans sa cavité un double interféromètre de Michelson [3] qui permet d'obtenir une puissance utile plus élevée (en raison des très faibles pertes énergétiques qu'il introduit) que celle permise par les sélecteurs de mode "étalons épais » de type Fabry-Perot couramment utilisés dans les modèles commerciaux (en particulier dans le cas du 580 A S.P.).

Actuellement, les lasers à colorant en anneau fournissent des puissances supérieures à celles des lasers avec double Michelson : toute l'étude que nous présentons ici sur la préstabilisation et le verrouillage de la fréquence sur une référence atomique s'applique également à ce type de laser.

1.1 RÉALISATION D'UN LASER MONOMODE PAR LA TECHNIQUE DU DOUBLE INTERFÉROMÈTRE DE MICHELSON. - Le laser à colorant continu traditionnel est constitué d'une cavité repliée à trois miroirs $\left(\mathrm{M}_{0}\right.$, $\mathrm{M}, \mathrm{M}_{1}$ ) conformément au schéma figure 1 , le milieu amplificateur formé d'un jet laminaire de colorant est excité par un laser à argon ionisé $\left({ }^{1}\right)$. Dans la cavité, d'intervalle spectral libre $375 \mathrm{MHz}$, est placé un premier élément sélectif (ici un filtre de Lyot) qui permet de choisir la longueur d'onde émise à l'intérieur de la zone spectrale autorisée par le colorant.

Pour obtenir une oscillation monomode nous avons remplacé le miroir de sortie par un double interféromètre de Michelson (Fig. 1) ce qui revient à réaliser trois cavités lasers de longueurs différentes $L_{1}, L_{2}, L_{3}$ ayant une partie commune. Les seules fréquences susceptibles d'osciller seront celles correspondant à un mode commun à chacune des trois cavités : $\mathrm{I}\left(\mathrm{M}_{0}, \mathrm{M}_{1}\right), \mathrm{II}\left(\mathrm{M}_{0}, \mathrm{M}_{2}\right), \mathrm{III}\left(\mathrm{M}_{0}, \mathrm{M}_{3}\right)$.

Nous avons choisi $L_{2}$ voisin de $L_{1}$ pour qu'il n'y ait pas plus d'une coïncidence entre les modes des cavités I et II dans la zone spectrale sélectionnée par le filtre de Lyot ( $\delta v_{\text {Lyot }}$ de l'ordre de 150 à $200 \mathrm{GHz}$ ) donc

$$
L_{1}-L_{2} \leqslant \frac{c}{2 \delta v_{\text {Lyot }}} .
$$

Le choix de $L_{1}-L_{2}$ dépend aussi de la puissance utile recherchée. Car, plus on augmente le gain, plus la plage de fréquence où le laser peut osciller augmente (gain $>$ pertes); nous avons alors réduit la plage de fonctionnement du filtre de Lyot en insérant dans la cavité un étalon de Fabry-Perot mince (lame de silice de 0,5 mm d'épaisseur aux faces non

( $\left.{ }^{1}\right)$ Dans notre cas, le colorant utilisé est une solution de Rhodamine $6 \mathrm{G}$ « R6G » dans l'éthylène glycol et le laser à argon émet sur la raie verte à $514,5 \mathrm{~nm}$. 


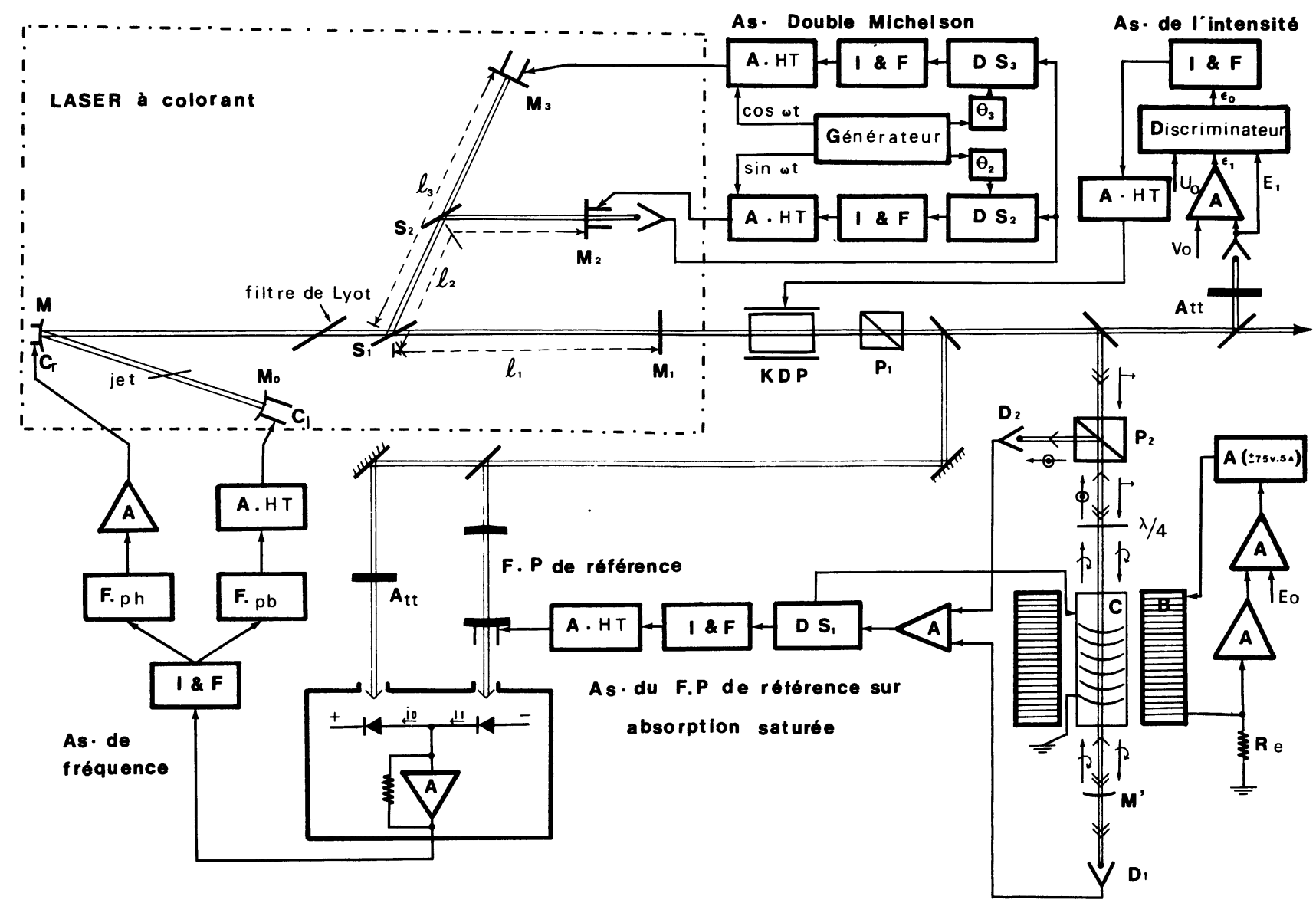

Fig. 1. - Schéma d'ensemble du laser à colorant stabilisé. Le laser est rendu monomode par l'interféromètre double Michelson constitué des séparatrices $S_{1}, S_{2}$ et des miroirs $M_{2}, M_{3}$ dont la position est contrôlée par deux boucles d'asservissement. La puissance émise est stabilisée par l'asservissement d'intensité agissant sur le cristal de KDP. La fréquence du laser est verrouillée sur le pic de transmission du Fabry-Perot de référence (F.P. réf.) à l'aide d'une double boucle d'asservissement pilotant une céramique lente $\left(C_{L}\right)$ et une céramique rapide $\left(C_{r}\right)$. Le Fabry-Perot de référence est lui-même contrôlé par un asservissement sur une raie d'absorption saturée du néon. Dans la cellule $(C)$ le néon est excité par une décharge continue, il est soumis à un champ magnétique (bobine B) qui assure l'excursion de la fréquence du pic d'absorption saturée. Une petite bobine enroulée directement sur la cellule crée une faible modulation de l'induction magnétique et permet la génération du signal d'erreur par la détection synchrone (D.S $)_{1}$.

Tous les asservissements utilisent un intégrateur et un filtre (I § F) comme élément correcteur et un amplificateur (A) qui adapte le niveau du signal à la commande de l'élément régulateur.

[General scheme of the stabilized dye laser. Monomode operation of the dye laser is obtained by the use of a double Michelson interferometer comprising two beam splitters $\left(S_{1}\right.$ and $\left.S_{2}\right)$ and two mirrors $\left(M_{2}\right.$ and $\left.M_{3}\right)$ the positions of which are controlled by two servo-loops. The emitted power is stabilized by the intensity control system acting on the KDP crystal. The laser frequency is locked on the transmission peak of the reference Fabry-Perot (F.P. ref.) with the help of a double servo-loop acting respectively on a slow PZT $\left(C_{L}\right)$ and on a fast PZT $\left(C_{r}\right)$. The reference Fabry-Perot is itself locked on a saturated absorption line of neon. In the cell (C), neon is excited by a DC discharge and submitted to a magnetic field (B coil) which insures the frequency scanning of a saturated absorption peak. A small coil produces a modulation of the magnetic field and a lock-in amplifier (D.S $S_{1}$ ) provides the error signal. All the servo-loops are constituted by an integrator and a filter (I § F) as a correcting element and by an amplifier (A) which adapts the level of the error signal for driving the regulating element.]

traitées) de façon à augmenter la puissance émise : la largeur spectrale est alors réduite à $50 \mathrm{GHz}$ ce qui autorise une différence $L_{1}-L_{2}$ de l'ordre de $3 \mathrm{~mm}$.

Cependant, une configuration à deux cavités (simple Michelson) I et II comme définies ci-dessus, est insuffisante pour assurer un fonctionnement correct monomode car plusieurs modes peuvent osciller autour de la fréquence de coïncidence. La troisième cavité, III, doit donc augmenter la sélectivité près de la coïncidence entre I et II. Nous avons choisi $L_{3}$ très différent de $L_{1}$ et de $L_{2}$. La valeur idéale de $L_{3}-L_{1}$ $\left(\simeq L_{3}-L_{2}\right)$ doit être déterminée en tenant compte du « hole burning spatial " provoqué par la saturation du milieu amplificateur par l'onde stationnaire. Si deux modes sont tels que les nouds de l'un correspondent aux ventres de l'autre il n'y a pas de saturation croisée entre ces deux modes et la saturation due à l'un ne gêne pas l'oscillation de l'autre; le laser aura donc tendance à osciller sur ces deux modes simultanément. Sachant que tous les modes ont un noud sur le miroir $\mathbf{M}_{0}$ et que la distance de $\mathbf{M}_{0}$ au jet de colorant est de $50 \mathrm{~mm}$, on peut montrer que si nous 
voulons faire osciller un mode donné, $v_{0}$, les modes sans interaction avec $v_{0}$ ont les fréquences

$$
v_{0}+(2 N+1) \times 1,5 \mathrm{GHz} .
$$

Une différence $L_{3}-L_{1}$ de $50 \mathrm{~mm}$ permet de rejeter tous les modes dangereux et assure une bonne sélectivité ( $25 \mathrm{~mm}$ est aussi une valeur acceptable).

La puissance obtenue est alors de $500 \mathrm{~mW}$ à $600 \mathrm{~nm}$, puissance bien supérieure à celle fournie par le laser 580 A S.P.

La configuration et l'interdépendance de ces éléments $\left(M_{0}, M_{1}, M_{2}, M_{3}\right)$ entraînent que toute modification ou perturbation sur l'un d'eux cause un saut de mode. Il est donc nécessaire d'obliger les cavités II et III à suivre le mode oscillant de la cavité I prise comme référence. Il suffit pour cela, d'asservir les longueurs $L_{2}$ et $L_{3}$ sur le pic de résonance de la cavité originale I à l'aide de deux servomécanismes.

1.2 AsservisSement du SÉleCteUR DE MODE (DOUBLE INTERFÉROMĖTRE DE MICHELSON) (FIG. 1). L'intensité du laser présente un maximum lorsque la fréquence

$$
v=\frac{N_{1} c}{2 L_{1}}=\frac{N_{2} c}{2 L_{2}}=\frac{N_{3} c}{2 L_{3}} .
$$

Si les bras du double interféromètre de Michelson s'écartent de cette position idéale, l'intensité décroît, on obligera donc les deux bras II et III à suivre les variations de la cavité I de façon à ce que l'intensité reste maximale.

Dans le cas d'une variable (simple Michelson ou étalon interne) l'asservissement sur le maximum est réalisé en modulant légèrement la longueur du bras (ou de l'étalon interne) à asservir puis en détectant la modulation résultante à l'aide d'une détection synchrone qui fournit un signal passant par zéro lorsque l'intensité est maximale (signal approximativement égal à la dérivée de l'intensité par rapport à la longueur du bras). On applique alors le signal obtenu, après traitement électronique approprié, à la céramique solidaire du miroir définissant la longueur de ce bras (ou de l'étalon interne). Dans le cas des deux variables (double Michelson) le calcul montre (Appendice I) que la variation de l'intensité est de la forme :

$$
I=I_{0}-\alpha\left(\Delta L_{2}^{2}+\Delta L_{3}^{2}-\Delta L_{2} \Delta L_{3}\right)
$$

où $\Delta L_{2}$ et $\Delta L_{3}$ sont les écarts aux positions idéales.

On peut appliquer la technique d'asservissement par détection synchrone aux deux bras $L_{2}$ et $L_{3}$ en modulant respectivement ces longueurs aux fréquences $f_{2}$ et $f_{3}$ et en corrigeant $L_{2}$ (et $L_{3}$ ) grâce au signal d'erreur obtenu à la fréquence $f_{2}$ (et $f_{3}$ ). Par cette technique, on ne peut découpler les corrections dues au terme croisé $\Delta L_{2} \Delta L_{3}$ et une erreur sur $L_{2}$ entraîne un signal de correction sur $L_{2}$, mais aussi sur $L_{3}$. Malgré cette interdépendance on peut montrer théoriquement que l'ensemble converge vers l'équi- libre recherché et, pratiquement, le système ainsi réalisé fonctionne correctement.

Cependant, nous avons mis au point une méthode qui permet de découpler les deux asservissements. Pour cela, comme nous le montrons dans l'appendice I, il suffit de moduler les deux bras $L_{2}, L_{3}$ avec deux signaux de même amplitude et de même fréquence mais déphasés de $\pi / 2$ puis de régler correctement les phases des détections synchrones. Il existe un réglage pour lequel les deux asservissements sont parfaitement désolidarisés. Expérimentalement on règle la phase $\theta_{2}$ de sorte que le signal sortant de la détection synchrone 2 ne soit plus sensible à une modulation lente de $L_{3}$ (et réciproquement pour $\theta_{3}$ ).

Cette deuxième méthode fonctionne correctement et l'asservissement ainsi réalisé semble nettement plus stable et plus facile à verrouiller que l'asservissement à boucles couplées.

Il est à noter que la fréquence maximale, $f_{\mathrm{f}}$, des fluctuations susceptibles d'être corrigées par cette technique d'asservissement (utilisant des détections synchrones) est pratiquement de l'ordre du dixième de la fréquence de modulation $f_{0}$. On a donc intérêt à choisir $f_{0}$ aussi grand que possible : ce choix est cependant limité par les fréquences de résonance des céramiques solidaires des miroirs $M_{2}$ et $M_{3}$ et pratiquement $f_{0}$ ne peut dépasser quelques kilohertz. La bande passante des asservissements est alors limitée à quelques centaines de hertz, mais ceci est largement suffisant pour assurer la stabilité de l'ensemble. En effet, les fluctuations importantes et rapides que peut présenter la fréquence du laser proviennent du milieu actif (jet) qui appartient à la partie commune aux trois cavités de telle sorte que les variations relatives des trois longueurs optiques $L_{1}, L_{2}, L_{3}$ restent faibles et faciles à corriger (ceci est encore un avantage du système double Michelson).

Nous disposons maintenant d'un faisceau qui oscille bien sur un seul mode mais dont la fréquence et l'intensité présentent encore des fluctuations : le «jitter» du laser libre est de 20 à $30 \mathrm{MHz}$ (cas du 590 C.R.) et les variations d'intensité $\left({ }^{2}\right)$ atteignent $20 \%$ du niveau moyen si l'on fait abstraction de certaines fluctuations de type "impulsionnel » provoquant parfois l'extinction du faisceau pendant quelques microsecondes (microbulles dans le jet). Notons également que l'asservissement du double Michelson par la méthode de modulation introduit une modulation de la fréquence et de l'intensité à la fréquence $f_{0}$ et qu'il nous faut aussi l'éliminer (c'est une raison pour ne pas prendre $f_{0}$ trop élevé).

Comme nous l'avons indiqué dans l'introduction, les fluctuations de fréquence ont un spectre beaucoup trop large pour être corrigées entièrement par l'asser-

$\left({ }^{2}\right)$ Ces variations d'intensité, anormalement fortes, proviennent du long trajet du faisceau pompe imposé, dans notre cas, par la nécessité de pomper deux lasers à colorant avec le même laser à argon. 
vissement sur le signal d'absorption saturée. Il nous faut donc préstabiliser la fréquence du laser sur un Fabry-Perot de référence, de plus il nous faut aussi stabiliser l'intensité du faisceau émis.

1.3 PrÉStabilisation dU LASER. - 1.3.1 Asservissement de la fréquence du faisceau émis. - La stabilisation en fréquence est obtenue en agissant directement sur la longueur de la cavité. On compare la fréquence d'émission laser à une fréquence de référence fournie par le pic de transmission d'un Fabry-Perot. Nous pourrions utiliser la méthode de modulation et asservir le laser au sommet du pic; cependant comme nous l'avons indiqué, la bande passante de l'asservissement est réduite au dixième de la fréquence de modulation, elle-même limitée par la résonance mécanique de la céramique piézo-électrique contrôlant soit la longueur du Fabry-Perot soit la fréquence du laser. Nous avons préféré l'asservissement à flanc du pic de transmission du FabryPerot; dans ce cas, les fluctuations de fréquence sont transformées en fluctuations d'intensité de la lumière transmise et pratiquement sans limitation de la fréquence (détecteurs photodiodes très rapides).

Pour définir le signal d'erreur, il suffit de comparer le signal fourni par une photodiode (placée après le Fabry-Perot) à une tension de référence $v_{0}$. Mais comme nous l'avons déjà indiqué, l'intensité du faisceau n'est pas constante et ses variations seront aussi interprétées comme des variations de fréquence. Pour pallier ce défaut, la solution la plus simple est de générer $v_{0}$ à l'aide d'un détecteur recevant une partie de l'intensité laser. Un atténuateur optique, de transmission réglable, permet d'ajuster le niveau $v_{0}$ désiré. Ainsi le signal d'erreur ne dépend plus de l'intensité du laser mais seulement des fluctuations de fréquence $\left({ }^{3}\right)$. Il est alors amplifié, mis en forme et commande une céramique piézo-électrique solidaire de l'un des miroirs de la cavité. En fait, une analyse des bruits de fréquence a montré l'existence d'une part de fluctuations lentes de grandes amplitudes et d'autre part de fluctuations beaucoup plus rapides mais de faibles amplitudes. Nous avons alors réalisé un asservissement «double boucle » $[4,5]$. Cet asservissement, à partir du même signal d'erreur, génère deux signaux correcteurs, qui, appliqués respectivement à deux céramiques de caractéristiques complémentaires (permettant, l'une de fortes élongations, l'autre de variations rapides) assurent la correction d'un maximum de fluctuations.

$\left({ }^{3}\right)$ Lors de la réalisation pratique du signal d'erreur, la grande difficulté fut d'obtenir la même réponse pour les deux détecteurs. Nous avons en particulier rejeté un premier montage où chaque détecteur avait son propre amplificateur : les microcoupures d'intensité du faisceau provoquaient des variations dramatiques du signal d'erreur. Il faut également que la différence $I-I_{0}$ soit correctement réalisée, ce qui n'est pas le cas avec un amplificateur différentiel (mauvaise symétrie entre les deux voies, erreur due à la tension en mode commun). En conséquence, nous avons adopté le montage figure 1 , dans lequel nous effectuons directement la somme algébrique des courants traversant les deux photodiodes.
Cet asservissement transfère, au mieux, à la fréquence d'émission les qualités de l'interféromètre Fabry-Perot de référence (cavité passive, mécaniquement stable mais sensible aux dérives thermiques). La limite d'efficacité est liée essentiellement, dans notre cas, à la résonance mécanique de la céramique piézo-électrique « rapide » $(49 \mathrm{kHz})$ : la correction des bruits de fréquence s'effectue jusqu'à environ $15 \mathrm{kHz}$.

Cet asservissement permet de plus un pilotage de la fréquence d'émission sur plusieurs gigahertz par action sur la longueur de la cavité Fabry-Perot.

\subsubsection{Asservissement de l'amplitude du faisceau} émis. - Les fluctuations que doit corriger l'asservissement sont de deux sortes, celles que nous dirons " traditionnelles", de faibles amplitudes et de large spectre de fréquence (quelques centaines de kilohertz) et celles déjà indiquées, de type «impulsionnel » provoquant des "trous » d'intensité de durée et de profondeur variable. Alors que les premières sont parfaitement corrigées par un asservissement linéaire classique, les secondes entraînent des instabilités de fonctionnement (trains d'oscillations). Pour remédier à ce défaut de fonctionnement nous avons réalisé un système logique (Appendice II) qui permet d'inhiber l'asservissement pendant la durée des impulsions de trop grande amplitude (nous avons depuis lors, éliminé la plus grande partie des microbulles d'air par filtrage correct de la solution de colorant). La stabilisation en amplitude est obtenue en comparant à chaque instant l'intensité du faisceau à une grandeur de référence $v_{0}$ puis en appliquant le signal d'erreur, amplifié, à un élément atténuateur électro-optique (deux cristaux de KDP suivi d'un prisme de Glan) qui contrôle ainsi l'intensité du faisceau.

Cet asservissement, eu égard au spectre de fréquence des perturbations, comporte uniquement des éléments de très larges bandes passantes : le détecteur $(10 \mathrm{MHz}$ à gain unité), le cristal KDP (100 MHz), les amplificateurs «45K Analog Devices » $(10 \mathrm{MHz}$ à gain unité)... De leurs qualités dépendent les performances de l'asservissement. Dans le montage réalisé, les fluctuations de fréquence inférieures ou égales à $100 \mathrm{kHz}$ sont parfaitement corrigées.

Performances obtenues. - Le laser ainsi préstabilisé présente encore des fluctuations d'amplitude de quelques centièmes aux fréquences supérieures à $100 \mathrm{kHz}$ et des fluctuations de fréquence de 2 à $3 \mathrm{MHz}$ d'amplitude crête à crête (soit mieux que $1 \mathrm{MHz}$ efficace) aux fréquences supérieures à $15 \mathrm{kHz}\left({ }^{4}\right)$. Ces performances sont comparables à celles des lasers commerciaux qui utilisent des techniques analogues. Elles peuvent être considérablement améliorées [6] en remplaçant tous les composants électroniques par des composants « rapides » (de bande passante au moins égale au gigahertz à gain unité) ainsi que la

$\left({ }^{4}\right)$ Avec le laser 580 A S.P. les performances obtenues sont deux fois meilleures mais avec quelques dizaines de milliwatts de puissance utile au lieu de quelques centaines. 
céramique rapide de l'asservissement de fréquence par un cristal électro-optique modulant la longueur optique de la cavité. Cependant nos expériences ne nécessitaient pas de telles améliorations onéreuses et difficiles à mettre en œuvre, par contre il nous fallait une référence absolue (corrigeant les dérives lentes du Fabry-Perot) largement balayable. C'est pourquoi nous avons réalisé le montage d'absorption saturée dans un champ magnétique réglable.

2. Asservissement sur la référence atomique balayable magnétiquement. - Toute la mise au point a été faite sur la raie du néon à $616,3 \mathrm{~nm}$, cette raie a une seule composante $\left(\sigma^{+}\left(\right.\right.$ou $\left.\left.\sigma^{-}\right)\right)$. Nous avons réalisé une expérience d'absorption saturée où le faisceau saturant et le faisceau sonde sont polarisés en $\sigma^{+}$(ou $\sigma^{-}$), en conséquence le pic d'absorption saturée peut être déplacé par effet Zeeman : nous disposons ainsi d'une référence balayable magnétiquement.

Le montage de l'expérience d'absorption saturée et l'asservissement sont décrits figure 1 . Le faisceau saturant $I_{\mathrm{sa}}$, prélevé par la séparatrice $\mathrm{S}$, traverse le prisme de Glan $\mathrm{P}_{2}$ qui lui assure une polarisation rectiligne verticale puis la lame quart d'onde le polarise circulairement (circulaire droite par rapport au sens de propagation, c'est-à-dire $\sigma^{+}$si le champ $B$ est orienté dans ce sens). Il traverse ensuite le milieu atomique et sature la transition choisie (raie à $616,3 \mathrm{~nm}$ du néon). Enfin la partie transmise par le miroir $\mathbf{M}^{\prime}$ est détectée par la photodiode $D_{1}$. Le miroir $\mathbf{M}^{\prime}$ de rayon de courbure adapté au front d'onde, réfléchit $4 \%$ de $I_{\mathrm{sa}}$ et fournit un faisceau sonde $I_{\mathrm{so}}$ polarisé lui aussi $\sigma^{+}$puisque le sens absolu de rotation du champ électrique de l'onde est conservé (la lumière devient circulaire gauche puisque son sens de propagation est inversé). Le faisceau sonde traverse le milieu atomique, sa polarisation est transformée en polarisation rectiligne horizontale par la lame quart d'onde : il est donc totalement réfléchi par le prisme de Glan vers le détecteur $D_{2}$. Cette configuration des éléments permet une superposition parfaite des deux faisceaux, elle évite tout retour dans le laser.

La cellule d'absorption est un tube, de $5 \mathrm{~mm}$ de diamètre, de $180 \mathrm{~mm}$ de longueur utile, fermé par des fenêtres traitées antireflet. Elle est remplie de néon excité par une décharge continue. La pression $(0,6$ à 2 torr) et le courant décharge ( 5 à $20 \mathrm{~mA}$ ) sont ajustés pour que la densité d'atomes métastables produits assure une absorption correcte des faisceaux lasers (pour certaines raies, la longueur de $180 \mathrm{~mm}$ s'avère trop grande car pour une décharge minimale l'absorption est presque totale).

Le signal d'absorption saturée, détecté par $D_{2}$, apparait sur un fond très important d'absorption linéaire. Ce fond est beaucoup plus large (en fréquence) que le pic d'absorption saturée, il n'affecte pas sa forme mais il introduit un bruit très important car son amplitude dépend de la décharge dans la cellule.
Nous avons amélioré le rapport signal sur bruit en retranchant au signal détecté par $D_{2}$ une partie de celui vu par $D_{1}$ (signal d'absorption linéaire pur entaché lui aussi du bruit dû à la décharge dans la cellule). La différence $D_{2}-\alpha D_{1}$ est équilibrée expérimentalement pour minimiser le bruit. Pour verrouiller la fréquence du laser sur le signal d'absorption saturée nous avons choisi de l'asservir au sommet du pic par la méthode de modulation. En effet, dans le cas présent, nous ne cherchons pas à obtenir une bande passante élevée (les fluctuations rapides ayant été corrigées par la préstabilisation sur Fabry-Perot), mais nous devons nous affranchir du bruit résiduel (encore important) sur le signal $D_{2}-\alpha D_{1}$. Or la technique par modulation et détection synchrone permet, en centrant le signal physique sur la fréquence $f_{0}$, de choisir un domaine de plus faible bruit que celui à fréquence nulle.

Nous avons réalisé une modulation (à $f_{0} \sim 7 \mathrm{kHz}$ ) de la position du pic d'absorption saturée en appliquant aux atomes un petit champ magnétique modulé à l'aide d'une bobine de très faible inductance enroulée directement sur la cellule. Le signal, fourni par la détection synchrone (qui est sensiblement la dérivée première du signal $D_{2}-\alpha D_{1}$ ), est utilisé comme signal d'erreur puis après intégration et amplification, il est appliqué à la céramique piézo-électrique contrôlant la longueur du Fabry-Perot de référence sur lequel est verrouillée la fréquence du laser.

Le positionnement ou le balayage de la raie atomique de référence par effet Zeeman est assuré par la commande du courant dans la bobine générant le champ d'induction magnétique appliqué à la cellule. Afin d'éviter tout élargissement de la raie en champ fort nous avons réalisé un champ magnétique homogène à quelques millièmes près sur toute la longueur utile $(18 \mathrm{~cm})$ de la cellule. Un tel champ est obtenu à partir d'un solénoïde, de longueur $(\sim 30 \mathrm{~cm})$ bien supérieure à celle de la cellule dont on a compensé les effets d'extrémités par des bobines complémentaires placées aux deux bouts. Pour balayer le champ symétriquement autour de zéro nous avons alimenté la bobine à l'aide d'un amplificateur opérationnel de puissance $( \pm 72 \mathrm{~V}, 5 \mathrm{~A})$. Nous obtenons ainsi un champ de $\pm 950 \mathrm{G}\left({ }^{5}\right)$ ce qui permet une excursion de la fréquence de $\pm 1,8 \mathrm{GHz}$ pour la raie à $616,3 \mathrm{~nm}$ $(g=1,34)$. La stabilité et la reproductibilité du champ (indispensables puisque le champ définit la fréquence du laser) ont été obtenu grâce à un asservissement du courant dans la bobine : le signal d'erreur est donné par la différence entre la tension aux bornes d'une résistance étalon $(0,01 \Omega)$ et une tension de référence balayable. Dans toutes nos expériences la tension aux bornes de la résistance étalon nous sert à mesurer la fréquence du laser. Utilisée comme signal $X$ d'un

$\left({ }^{5}\right)$ Pour le second laser, nous avons réalisé une bobine de plus petit diamètre, elle nous permet d'obtenir avec la même alimentation $\pm 1400 \mathrm{G}$, soit une excursion de $\pm 2,6 \mathrm{GHz}$. 
enregistreur $X Y$ elle nous permet d'enregistrer des spectres très commodément.

Lors d'une expérience d'absorption saturée à deux lasers [7], l'un fournissant le faisceau saturant de fréquence fixe, l'autre le faisceau sonde de fréquence balayable, nous n'avons pas observé de dérive appréciable de la position de la résonance durant plusieurs heures. Etant donné la largeur du signal dans ces expériences nous pouvons estimer la reproductibilité du balayage meilleure que 1 mégahertz. Ceci correspond à environ $4 \%$ de la largeur de la raie de référence $\left({ }^{6}\right)$ et à une stabilité du champ magnétique de $1 \mathrm{G}$ soit $10^{-3}$ de la valeur maximale du champ : les performances de notre laser sont certainement supérieures à ces valeurs mais nous n'avons pas pu les mesurer.

Appendice I. - Nous présentons dans cet appendice un calcul de l'intensité émise par le laser lorsque les longueurs des bras du double interféromètre de Michelson sont légèrement déréglées par rapport à leur valeur idéale et nous en déduisons la méthode d'asservissement.

En supposant que les coefficients de transmission des séparatrices $S_{1}, S_{2}$ et du miroir de sortie $M_{1}\left(T_{1}\right)$ sont tels que les trois ondes réfléchies aient exactement la même amplitude, on peut considérer le double Michelson comme un seul miroir de coefficient de réflexion complexe :

$$
R=\frac{1}{3}\left(1-\frac{T_{1}}{6}\right) \sum_{i=1}^{3} \exp \left(-\frac{j 4 \pi v l_{i}}{c}\right)
$$

L'équation d'équilibre de l'oscillation laser à la fréquence $v$ s'écrit :

$$
R \exp \left(-\frac{j 4 \pi v L_{0}}{c}\right) G(I) r_{0}=1
$$

où

- $G(I)$ est le gain saturé du milieu amplificateur,

- $L_{0}$ est la longueur optique (éventuellement saturée) de la partie commune aux trois bras,

- $r_{0}$ est le coefficient de réflexion du miroir $\mathbf{M}_{0}$ (où sont supposées rassemblées toutes les pertes).

La partie imaginaire est :

$$
\sum_{i=1}^{3} \sin \varphi_{i}=0
$$

la partie réelle :

$$
\frac{1}{3}\left(1+\frac{T_{1}}{6}\right) r_{0} G(I) \sum_{i=1}^{3} \cos \varphi_{i}=1
$$

$\left.{ }^{6}{ }^{6}\right)$ La largeur des pics d'absorption saturée de références était de l'ordre de $50 \mathrm{MHz}$, à pression et à intensité laser plus faibles nous pouvions réduire de moitié cette largeur de raie. avec

$$
\varphi_{i}=\frac{4 \pi v}{c}\left(l_{i}+L_{0}\right)=\frac{4 \pi v}{c} L_{i} .
$$

La relation (1) définit la fréquence du mode pour des $L_{i}$ quelconques, tandis que la relation (2) définit son intensité de façon implicite.

L'accord du double Michelson est optimal et l'intensité maximale $\left(I_{\mathrm{M}}\right)$ lorsque :

$$
\sum_{i=1}^{3} \cos \varphi_{i}=3
$$

soit

$$
v=N_{1} \frac{c}{2 L_{1}}=N_{2} \frac{c}{2 L_{2}}=N_{3} \frac{c}{2 L_{3}} .
$$

Si cette condition n'est pas réalisée, le mode qui en est le plus proche (c'est-à-dire pour lequel $\sum_{i=1}^{3} \cos \varphi_{i}$ est maximal, (1) étant réalisé) oscille mais son intensité n'est pas maximale.

Supposons que $L_{1}, L_{2}$ et $L_{3}$ s'écartent légèrement de la position idéale (condition (3)). En différentiant l'équation (2) (au premier ordre par rapport à $I$, au second ordre par rapport aux $\varphi_{i}-$ car le premier ordre est nul -) on obtient :

$$
3 G^{\prime}\left(I_{\mathrm{M}}\right) \Delta I-\frac{1}{2} G\left(I_{\mathrm{M}}\right) \sum_{i=1}^{3}\left(\Delta \varphi_{i}\right)^{2}=0
$$

avec

$$
\Delta \varphi_{i}=\frac{4 \pi}{c}\left(L_{i} \Delta v+v \Delta L_{i}\right)
$$

La condition (1) s'écrit :

$$
\sum_{i=1}^{3} \Delta \varphi_{i}=0
$$

soit

$$
\Delta v \sum_{i=1}^{3} L_{i}=-v \sum_{i=1}^{3} \Delta L_{i} .
$$

Les conditions supplémentaires dépendent du mode de fonctionnement du laser. Si sa fréquence est asservie par l'action d'une céramique piézo-électrique solidaire du miroir commun $\mathbf{M}_{0}$, alors :

$$
\begin{aligned}
\Delta L_{1} & =\Delta L_{0} ; \Delta L_{2,3}=\Delta L_{0}+\Delta l_{2,3} ; \\
\Delta r & =0 \Rightarrow 3 \Delta L_{0}+\Delta l_{2}+\Delta l_{3}=0
\end{aligned}
$$

la relation (4) s'écrit :

$$
\Delta I=\frac{G}{9 G^{\prime}}\left(\frac{4 \pi v}{c}\right)^{2}\left[\left(\Delta l_{2}\right)^{2}+\left(\Delta l_{3}\right)^{3}-\Delta l_{2} \Delta l_{3}\right] .
$$

Lorsque le laser est libre $\left(\Delta L_{0}=0, \Delta v\right.$ donné par (5)), on retrouve approximativement la même expression. $G^{\prime}(I)$ étant négatif, $\Delta I$ est négatif quels que soient $\Delta l_{1}$ et $\Delta l_{2}$ (I maximal). 
AsSERVISSEMENT DU DOUBLE Michelson. - $\mathrm{Si}$ l'on module $l_{2}$ et $l_{3}$ respectivement aux fréquences $\omega_{2}$ et $\omega_{3}\left(\Delta l_{2,3}=\delta l_{2,3}+\beta \cos \omega_{2,3} t\right)$ le signal détecté sur l'intensité à ces fréquences est :

$$
\Delta I=\varepsilon_{2} \cos \omega_{2} t+\varepsilon_{3} \cos \omega_{3} t
$$

soit

$$
\Delta I=\alpha\left(2 \delta l_{2}-\delta l_{3}\right) \cos \omega_{2} t-\alpha\left(2 \delta l_{3}-\delta l_{2}\right) \cos \omega_{3} t .
$$

Le déplacement $\delta l_{3}$ du miroir $\mathrm{M}_{3}$ réagit sur le signal d'erreur $\varepsilon_{2}$ à la fréquence $\omega_{3}$ et réciproquement. $\delta l_{2}$ (ou $\delta l_{3}$ ) est dû aux perturbations extérieures $\mu_{2}$ (ou $\mu_{3}$ ) et au signal de correction $-T \varepsilon_{2}$ (ou $-T \varepsilon_{3}$ ) où $T$ est la fonction de transfert de l'asservissement. On obtient ainsi le système :

$$
\delta l_{2,3}=\mu_{2,3}-\alpha T\left(2 \delta l_{2,3}-\delta l_{3,2}\right)
$$

dont la solution est (lorsque $\alpha T \gg 1$ )

$$
\delta l_{2,3}=\left(\frac{2}{3} \mu_{2,3}+\frac{1}{3} \mu_{3,2}\right) \frac{1}{\alpha T} .
$$

Lorsque le gain $T$ des deux asservissements est très grand, $\delta l_{2}$ et $\delta l_{3}$ tendent vers zéro et l'asservissement est possible malgré le terme de couplage. Ce couplage complique beaucoup l'analyse du comportement dynamique de l'asservissement. Pratiquement, il est nécessaire de bien régler manuellement $\delta l_{2}$ et $\delta l_{3}$ (pour être proche des positions idéales) avant de verrouiller les asservissements.

En conséquence nous avons réalisé des asservissements découplés en modulant les deux bras du double Michelson avec deux signaux de même fréquence mais déphasés de $\pi / 2$.

Dans ce cas :

$$
\begin{aligned}
\Delta I(\omega) & =\alpha\left[\cos \omega t\left(2 \delta l_{2}-\delta l_{3}\right)+\sin \omega t\left(2 \delta l_{3}-\delta l_{2}\right)\right] \\
& =\alpha \sqrt{5}\left[\delta l_{2} \cos \left(\omega t+\theta_{2}\right)-\delta l_{3} \cos \left(\omega t+\theta_{3}\right)\right]
\end{aligned}
$$

avec

$$
\operatorname{tg} \theta_{2}=\frac{1}{2} \quad \text { et } \operatorname{tg} \theta_{3}=2 \text {. }
$$

Si l'on choisit la phase $\left(\theta_{2}+\pi / 2\right)$ pour l'une des détections synchrones on obtient un signal d'erreur $\varepsilon_{3}$ ne dépendant que de $\delta l_{3}$. En choisissant la phase $\left(\theta_{3}+\pi / 2\right)$ pour la deuxième détection synchrone on obtient $\varepsilon_{2} \propto \delta l_{2}$. Pratiquement ce réglage des phases est très facile, pour cela on module lentement $l_{2}$ (ou $l_{3}$ ) et l'on règle la phase de la détection synchrone 3 (ou 2) pour annuler l'effet de cette modulation.

Appendice II. - Les bulles d'air qui circulent dans le jet de colorant produisent des microcoupures de l'intensité du laser, elles se traduisent par des variations de type impulsionnel sur le signal d'erreur et entraînent un fonctionnement non linéaire (saturation, trains d'oscillations) de la boucle d'asservis- sement de l'amplitude du faisceau laser. Pour éviter ce comportement néfaste, le «discriminateur de bulles » substitue au signal d'erreur $\varepsilon_{1}$ un signal nul lorsque l'intensité $\left(E_{1}\right)$ du faisceau devient inférieur à une valeur seuil $\left(U_{0}\right)$ choisie égale à environ $90 \%$ de la valeur moyenne $\left(V_{0}\right)$ imposée par la consigne de l'asservissement. Ceci est réalisé par deux relais électroniques (CD 4016) commandés par un système logique (A ouvert et $\mathrm{B}$ fermé imposent $\varepsilon_{0}=0$, Fig. II. 1).

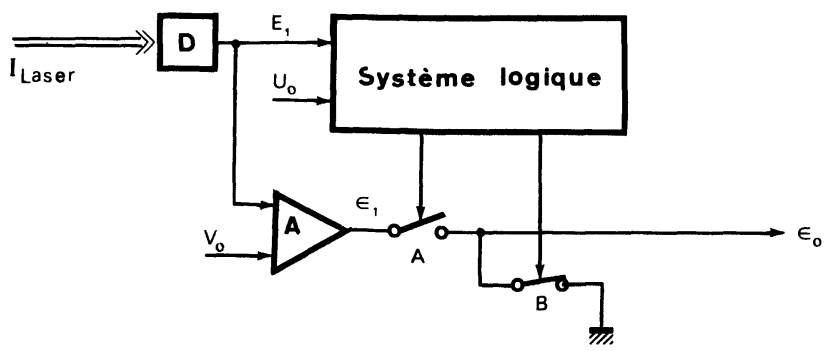

Fig. II.1. - Principe du discriminateur de bulles.

[Principle of the bubble discriminator.]

Le système logique, décrit figure II.2, est constitué d'un comparateur (SN 72710) qui transforme l'impulsion parasite en une impulsion normalisée de même largeur, de trois multivibrateurs monostables suivis d'un circuit (SN 7407) qui adaptent l'impulsion normalisée à la commande des relais électroniques (CD 4016).

Pour éviter le passage d'une partie de l'impulsion parasite dans le signal d'erreur, il est important que les relais agissent pendant un temps qui encadre correctement l'impulsion à éliminer. Pour le début de l'impulsion il n'y a aucune difficulté car les circuits logiques répondent plus vite que l'amplificateur différentiel (circuit analogique); lorsque la perturbation arrive sur le signal d'erreur $\left(\varepsilon_{1}\right)$, les relais sont déjà en position (A ouvert, $\mathrm{B}$ fermé). Par contre, à la fin de l'impulsion, il est nécessaire de prolonger le signal logique d'environ 30 ns (rôle du monostable (3)) avant de positionner à nouveau les relais pour transmettre le signal d'erreur $\left(\varepsilon_{1}\right)$.

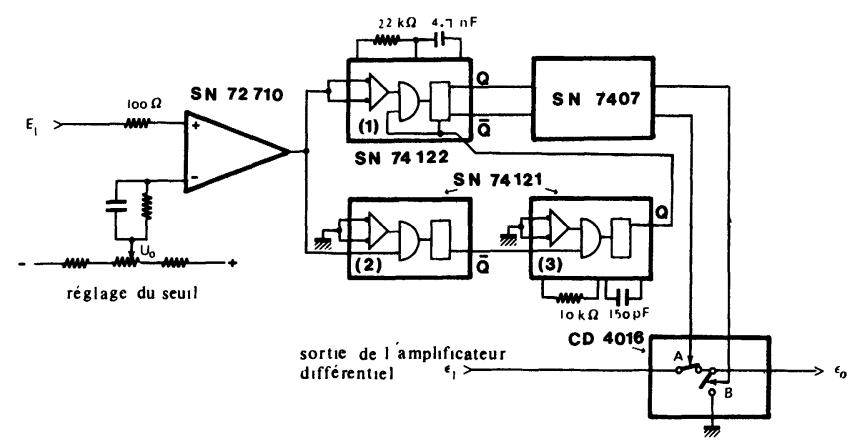

Fig. II.2. - Le discriminateur de bulles.

[The bubble discriminator.] 
Le « discriminateur de bulles » donne de très bons résultats : en augmentant progressivement le seuil de référence $\left(U_{0}\right)$, on voit disparaitre le train d'oscillations qui suit chaque microcoupure observée sur l'intensité du faisceau lorsque le gain de l'asservissement est élevé. Le bon réglage est obtenu pour $0,8 E<U_{0}<0,9 E$. Si $U_{0}$ est trop élevé, la moindre perturbation déclenche le discriminateur et l'asservissement ne joue plus son rôle de régulateur. Si $U_{0}$ est trop faible, l'asservissement est instable.

A la sortie du régulateur d'intensité (KDP) le faisceau laser présente toujours des microcoupures puisque nous ne pouvons pas les corriger, mais il retrouve son niveau stabilisé dès la fin de ce type de perturbation.

De plus «le discriminateur de bulles" peut permettre d'éliminer des points de mesure perturbés lors d'étude systématique au cours d'une expérience.

Appendice III. - Les divers asservissements réalisés pour stabiliser nos lasers à colorant tant en fréquence qu'en intensité sont des asservissements linéaires qui dérivent d'un même principe. Ils sont tels que leur fonction de transfert en boucle ouverte présente une réponse en amplitude dont la courbe asymptotique a une pente de $30 \mathrm{~dB} /$ décade sur la " plus large bande de fréquence " : à une pente de $30 \mathrm{~dB} /$ décade il correspond une expression mathématique de la forme $\frac{K_{0}}{\left(1+j \frac{\omega}{\omega_{1}}\right)^{3 / 2}}$, et un retard de phase maximal de $135^{\circ}$, quel que soit le gain $K_{0}$.

Ce type d'asservissement $[8,9]$ est inconditionnellement stable, jusqu'à des fréquences élevées, dans la limite de fonctionnement correct de chacun des éléments de la boucle.

Nous avons repris le principe général du correcteur mis au point par J. Hall. Ce correcteur est couramment utilisé dans les asservissements des lasers. Il a deux fonctions essentielles : il doit d'une part adapter le signal d'erreur à la commande de l'élément régulateur (céramique piézo-électrique, KDP) et d'autre part être tel que la fonction de transfert en boucle ouverte de l'asservissement présente la caractéristique définie précédemment.

Ce correcteur est constitué de trois sous-ensembles (Fig. III.1)

- un intégrateur de fonction de transfert $\left(T_{\mathrm{i}}\right)$,

- un filtre $\left(T_{\mathrm{f}}\right)$,

- un amplificateur haute-tension $\left(T_{\mathrm{a}}\right)$.

Le calcul des fonctions de transfert respectives de ces trois circuits donne :

- pour l'intégrateur :

$$
T_{\mathrm{i}}(j f)=\frac{U_{\mathrm{i}}}{U_{\mathrm{e}}}=\frac{Z_{2}}{R}=\alpha_{\mathrm{i}} \frac{1+j \frac{f}{f_{\mathrm{i} 12}}}{\left(1+j \frac{f}{f_{\mathrm{i} 1}}\right)\left(1+j \frac{f}{f_{\mathrm{i} 2}}\right)}
$$

avec

$$
\begin{aligned}
\alpha_{\mathrm{i}} & =\frac{R_{1}+R_{2}}{R} \\
f_{\mathrm{i} 1} & =\left(2 \pi R_{1} C_{1}\right)^{-1} \\
f_{\mathrm{i} 2} & =\left(2 \pi R_{2} C_{2}\right)^{-1} \\
f_{\mathrm{i} 12} & =\left[2 \pi \frac{R_{1} R_{2}}{R_{1}+R_{2}}\left(C_{1}+C_{2}\right)\right]^{-1}
\end{aligned}
$$

- pour le filtre :

$$
T_{\mathrm{f}}(j f)=\frac{\rho_{\mathrm{i}}^{\prime}}{R_{\mathrm{i}}} \frac{1}{1+\sum_{n} j \frac{f}{f_{\mathrm{f} n}^{\prime}}\left(1+j \frac{f}{f_{\mathrm{f} n}}\right)^{-1}}
$$

avec

$$
\begin{aligned}
\rho_{\mathrm{i}}^{\prime} & =\frac{\rho_{\mathrm{i}} \rho}{\rho_{\mathrm{i}}+\rho} \\
f_{\mathrm{in}}^{\prime} & =\left(2 \pi \rho_{\mathrm{i}}^{\prime} c_{n}\right)^{-1} \\
f_{\mathrm{f} n} & =\left(2 \pi r_{n} c_{n}\right)^{-1} .
\end{aligned}
$$

Le choix des couples $r_{n}, c_{n}\left(r_{n} c_{n}=10 r_{n+1} \cdot c_{n+1}\right)$ est tel que la réponse en amplitude dans l'intervalle de fréquences définies par $f_{\mathrm{f} 1}=\left(2 \pi r_{1} c_{1}\right)^{-1}$, $f_{\mathrm{f} 7}^{\prime \prime}=\left[2 \pi\left(\rho+r_{7}\right) c_{7}\right]^{-1}$ présente une pente de $-10 \mathrm{~dB} /$ décade lorsque le gain est maximal $\left(\rho_{\mathrm{i}}\right.$ est infini).

- pour l'amplificateur :

$$
T_{\mathrm{a}}(j f)=\frac{R_{2}}{R_{1}} \frac{1}{1+j \frac{f}{f_{\mathrm{a}}}}
$$

avec

$$
f_{\mathrm{a}}=\left(2 \pi R_{2} C_{2}\right)^{-1} \text {. }
$$

Dans la représentation de Bode, les réponses en amplitude et en phase du correcteur sont les sommes respectives des courbes élémentaires d'amplitude et de phase des trois circuits.

$$
\begin{aligned}
20 \log |H(j f)| & =20 \log \left(\left|T_{\mathrm{i}}\right| \cdot\left|T_{\mathrm{f}}\right| \cdot\left|T_{\mathrm{a}}\right|\right) \\
\operatorname{Arg} H(j f) & =\operatorname{Arg}\left(T_{\mathrm{i}} \cdot T_{\mathrm{f}} \cdot T_{\mathrm{a}}\right) .
\end{aligned}
$$

Les courbes de réponse en amplitudes des trois circuits du correcteur sont données figure III.1. La réponse globale présente alors une pente de - $30 \mathrm{~dB} /$ décade dans l'intervalle de fréquences compris entre $10^{-3} \mathrm{~Hz}$ et $2 \times 10^{3} \mathrm{~Hz}$, puis pour les fréquences supérieures la pente est de $-20 \mathrm{~dB} /$ décade et au-delà de $1,5 \mathrm{MHz}$ elle passe à $-40 \mathrm{~dB} /$ décade.

Pour définir la fréquence à gain unité en boucle ouverte et la stabilité du système, il faut connaître les autres éléments de la boucle. Si ceux-ci ont une réponse parfaitement plate quelle que soit la fréquence, il suffit de translater verticalement le diagramme d'amplitude, on peut ainsi augmenter le gain jusqu'à 


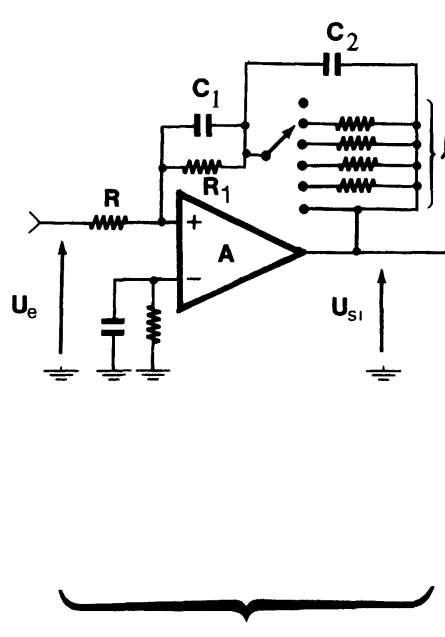

I NTE G RATEUR

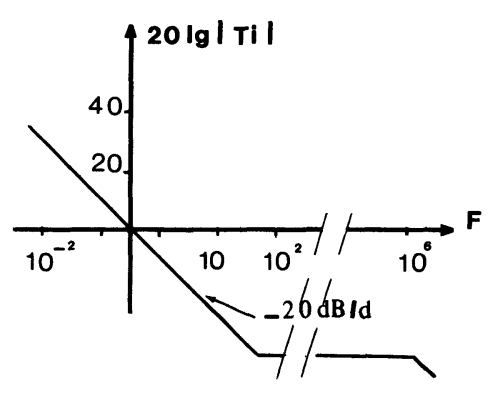

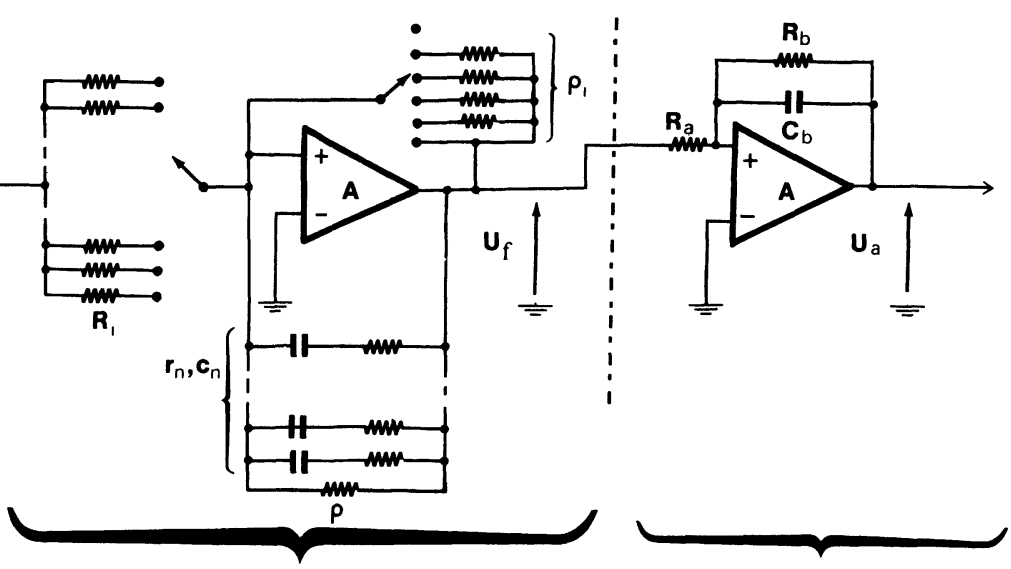

FILTRE

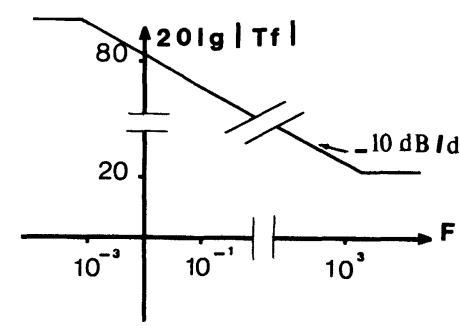

Fig. III. 1. ce que la fréquence à gain unité soit de $1,5 \mathrm{MHz}$ tout en conservant une marge de phase de $45^{\circ}$. Cette hypothèse tout à fait optimiste ne tient nullement compte des défauts de réponse des divers éléments vers les fréquences élevées et seule l'expérience permet de définir précisément le gain optimal assurant la rapidité optimale de réponse aux perturbations tout en conservant une bonne stabilité à l'asservissement.

\section{Bibliographie}

[1] Ouhayoun, M., Thèse, Docteur ès Sciences, Paris XIII (1979); Man ${ }_{2}$ C. N., Cérez, P., Brillet, A. and HartmanN, F., J. Physique Lett. 38 (1977) L-287.

[2] Camy, G., Thèse Ingénieur, Paris XIII (1979).

[3] Pinard, M., Aminoff, C. G. and Laloe, F., Appl. Phys. 15 (1978) $371-375$.

[4] Barger, R. L., Sorem, M. S. and Hall, J. L., Appl. Phys. Lett. 22 (1973) 573.
[5] Gardissat, J. L., Thèse C.N.A.M., Paris XIII (1976)

[6] Barger, R. L., West, J. B. and English, T. C., Appl. Phys. Lett. 27 (1975) 31-33.

[7] Gorlicki, M., Peuriot, A. and Dumont, M., J. Physique Lett. 41 (1980) L-275-L-278.

[8] Oustaloup, A., Onde Electrique 59, no 2 (1979) 41-49.

[9] Oustaloup, A., Onde Electrique 61, no 3 (1979) 31-37 et autres références citées dans cet article. 